\title{
Rayleigh-Ritz optimization of the structure function for a Kolmogorov power density and an approximate analytical solution for the two-point electric field correlation function
}

\author{
Monish R. Chatterjee, Lauren H. Quinn, Partha P. Banerjee, and Ting-Chung Poon
}

\begin{abstract}
Beginning with the exact Kolmogorov power spectrum for an extended random medium (such as interstellar space), the structure function is first expressed as an inverse Fourier-Bessel transform. An approximate series representation involving fractional powers of the transverse coordinate is then optimized by an equivalent Gaussian expansion up to two terms using the Rayleigh-Ritz technique. The domains of validity as well as the coherence profile of the resulting analytical solution of the two-point electric field correlation function are examined. It is shown that the correlation in both the transverse and longitudinal directions persists over distances up to a few orders of magnitude greater than that for a medium described by a Gaussian structure function.
\end{abstract}

\section{Introduction}

The propagation of optical or radio waves through random turbulent media has been studied for various physical systems. ${ }^{1-8}$ These have included weak and strong stellar scintillations, plasma fluctuations, source and image jitters, atmospheric turbulence, and sonar transmissions. The origin of the medium randomness may be associated with various factors, namely, electron density and refractive index variations, variations in atmospheric constituents, and aerosols.

The study of such propagation problems normally involves deriving and solving a differential equation for the amplitude correlation function or the intensity covariance function of the electric field. While much numerical studies have gone into the understanding of both, it turns out that finding exact analytical solutions often remains a prohibitive task. Lerche ${ }^{9} \mathrm{ob}-$ tained a closed solution for the two-point amplitude correlation at two frequencies by assuming a Gaussian

Ting-Chung Poon is with Virginia Polytechnic Institute \& State University, Bradley Department of Electrical Engineering, Blacksburg, Virginia 24061; P. P. Banerjee is with Syracuse University, Department of Electrical Engineering, Syracuse, New York 132441240; and the other authors are with SUNY at Binghamton, Department of Electrical Engineering, Binghamton, New York 13905.

Received 8 September 1988.

0003-6935/89/101773-00\$02.00/0.

(C) 1989 Optical Society of America. power spectrum and a corresponding Gaussian structure function. Such a Gaussian description is an oversimplification of the realistic power spectra (which, for astrophysical scintillations in particular, are more closely described by a Kolmogorov density); moreover, to obtain an analytical solution, it was necessary to expand the Gaussian structure up to the quadratic term only, thereby restricting the valid range of the solution in the transverse direction. Lee and Jokipii ${ }^{10-12}$ obtained numerical solutions in the so-called intermediate range; however, their series expansion of the structure function around the transverse coordinate $\rho=0$ appears to be invalid for a power law spectrum.

We start from the Kolmogorov power spectral density function and express the medium structure function in terms of a Fourier-Bessel transform. To obtain an analytical solution of the resulting integral, the Gaussian term in the integrand is then expanded to two terms (thereby restricting the region of validity to $\rho>$ $l$, where $l$ is the inner microscale of fluctuations). This leads to a series expansion up to $\rho^{2}$, including fractional powers of $\rho$. To use this structure to solve for the electric field correlation in a closed form, we apply a Rayleigh-Ritz optimization to express it as an expanded Gaussian in a preselected range. The final analytical solution, therefore, has the same form as in Lerche, ${ }^{9}$ with the exception that the range of applicability and the equivalent scale lengths are quite different from the Gaussian case.

In Sec. II, we briefly discuss the Lerche and Lee and Jokipii methods and their limitations as a prelude to 
our own approach. The derivation of the approximate structure function and its equivalent expanded Gaussian representation by the Rayleigh-Ritz technique are presented in Sec. III. An analytical solution for the two-point two-frequency electric field correlation, its domain of validity, and other implications are also discussed in this section. Section IV concludes this paper.

\section{Truncated Gaussian and Intermediate Range Methods}

A. Gaussian Power Spectrum and Approximate Analytical Solution for $\Gamma_{11}$

Following Lerche, ${ }^{9}$ we assume a medium with a Gaussian power spectral density

$$
P_{G}(q)=B_{G} \exp \left(-q^{2} / q_{G}^{2}\right),
$$

where $L_{G}=2 / q_{G}$ is the coherence scale of the fluctuations, and $B_{G}$ is a constant given by ${ }^{12}$

$$
B_{G}=128 \pi^{7 / 2}\left(r_{e}^{2} / k^{4} q_{G}^{3}\right)\left\langle\delta N_{e}^{2}\right\rangle,
$$

where $r_{e}=e^{2} / m c^{2}$ is the classical electron radius, $\left\langle\delta N_{e}^{2}\right\rangle$ is the mean-squared electron density fluctuations, and $k$ is the wavenumber.

Using Eq. (1), one can readily obtain the medium structure function

$$
A_{G}(\rho)=\left(B_{G} k^{4} q_{G}^{2} / 4 \pi\right) \exp \left(-\rho^{2} q_{G}^{2} / 4\right) .
$$

Next, one defines the two-point two-frequency electric field correlation as

$$
\Gamma_{11}\left(z, \rho, k_{1}, k_{2}\right) \equiv\left\langle E\left(z, r_{1}, k_{1}\right) E^{*}\left(z, r_{1}+\rho, k_{2}\right)\right\rangle,
$$

where $k_{1}$ and $k_{2}$ are the wavenumbers corresponding to two monochromatic waves originating from two points in the transverse domain, and * denotes the complex conjugate of the field amplitude. With the definition

$$
\Gamma_{11}=\exp \left[-z A_{G}(0)(1-R)^{2} / 4 k_{1}^{2}\right] \Gamma_{D}\left(z, \rho, k_{1}, k_{2}\right),
$$

where $R=k_{1} / k_{2}$, the relevant differential equation, under a quasioptical or parabolic approximation, becomes

$$
\begin{aligned}
\partial \Gamma_{D} / \partial z= & j\left[(1-R) / 2 k_{1}\right] \nabla_{\rho}^{2} \Gamma_{D} \\
& -\left[R A_{G}(0) / 2 k_{1}^{2}\right][1-a(\rho)] \Gamma_{D},
\end{aligned}
$$

where $a(\rho)=A_{G}(\rho) / A_{G}(0)$ is the normalized structure function, and $\nabla_{\rho}^{2}$ is the transverse Laplacian operator.

An expansion of $[1-a(\rho)]$ from Eq. (3) so that

$$
1-a(\rho) \cong \rho^{2} / L_{G}^{2},
$$

leads to an analytical solution for $\Gamma_{D}$ in Eq. (6) given by $^{9}$

$$
\begin{aligned}
\Gamma_{D}(\eta, \xi)= & \sec [2 \eta \exp (j \pi / 4)] \exp \left\{-0.5 \xi^{2}\right. \\
& \times \exp (-j \pi / 4) \tan [2 \eta \exp (j \pi / 4)]\},
\end{aligned}
$$

where $\eta$ and $\xi$ are normalized $\rho$ and $z$ variables, respectively, given by

$$
\begin{aligned}
& \eta=z\left(2 k_{1} L_{G}\right)^{-1}\left[R(R-1) A_{G}(0)\right]^{1 / 2} k_{1}^{-1 / 2}, \\
& \xi=\rho\left\{k_{1} L_{G}^{2}(R-1)\left[R A_{G}(0)\right]^{-1}\right\}^{-1 / 4} .
\end{aligned}
$$

The solution (8) may be readily derived by substituting

$$
\Gamma_{D}(\eta, \xi)=C(\eta) D\left[\xi^{2} / M(\eta)\right]
$$

into the approximated and normalized form of Eq. (6).

The expansion in Eq. (7) indicates that Eq. (8) is valid only in the range $0 \leq \rho \ll L_{G}$, where for strong interstellar scintillations $L_{G}$ is between $10^{14}$ and $10^{15}$ $\mathrm{cm}$. It turns out that Lee and Jokipii ${ }^{11}$ basically studied the same equation as Eq. (6) in the Gaussian case, but with the additional restriction that $|1-R| \ll 1$, which essentially means that the two frequencies involved in the second moment are virtually degenerate. Lerche's derivation indicates that such a restriction on $R$ is in fact not necessary.

\section{B. Kolmogorov Spectrum: Power Law Approximation}

Lee and Jokipii ${ }^{10-12}$ have studied the angular and temporal broadening of pulsar signals as well as intensity fluctuations (the fourth moment $\Gamma_{22}$ ) by assuming a Kolmogorov power density. The medium was assumed to be extended (more realistic than a thin screen model). The fluctuation in the refractive index was given a Markov approximation (Tatarski ${ }^{1}$ ). It was also shown that the strong scintillation problem was not always reducible to the far field approximation (in fact, in many cases, $z \ll L^{2} / \lambda$ ), so that Mercier's formula ${ }^{11}$ for a Gaussian spectrum in the Fraunhofer region was not applicable.

In their approach, Lee and Jokipii begin with the modified power law spectrum

$$
P_{K}(q)=\frac{B_{K} \exp \left(-q^{2} / q_{1}^{2}\right)}{\left(1+q^{2} / q_{0}^{2}\right)^{\alpha / 2}}
$$

which is Kolmogorov when $\alpha=11 / 3$. In Eq. (11), $B_{K}$ is a constant which is given by

$$
B_{K}=128 \pi^{7 / 2}\left(r_{e}^{2} / k^{4} q_{0}^{3}\right)\left\langle\delta N_{e}^{2}\right\rangle[\Gamma(\alpha / 2) / \Gamma(\alpha / 2-3 / 2)], \quad \alpha>3,
$$

and $l=1 / q_{1}$ is defined as the inner scale of fluctuations, while $L_{K}=1 / q_{0}$ is the coherence scale. In general, $q_{0}$ $\ll q_{1}$, and the spectrum in Eq. (11) has an approximate shape as shown in Fig. 1 . Typically, $l$ and $L_{K}$ lie in the $10^{5}-10^{7}$ - and $10^{17}-10^{21}$-cm ranges, respectively.

For more realistic results than the Gaussian case, Lee and Jokipii then assume a power law approximation of Eq. (11) so that for $q \ll q_{1}$,

$$
P_{K}(q) \cong B_{K}\left(1+q^{2} / q_{0}^{2}\right)^{-\alpha / 2} \text {. }
$$

Equation (13) leads to an exact structure function $A_{K}(\rho)$, which, by restricting $\rho$ to the intermediate range $l \ll \rho \ll L_{K}$, may be shown to be

$$
\begin{aligned}
A_{K}(\rho) \sim & \left\{\left(B_{K} q_{0}^{2} k^{4}\right) /[4 \pi(\alpha-2)]\right\} \\
& \times\left\{1-[\Gamma(2-\alpha / 2) / \Gamma(\alpha / 2)]\left(q_{0} \rho / 2\right)^{\alpha-2}\right\}, \quad \alpha>3 .
\end{aligned}
$$

However, at this point, Lee and Jokipii attempt to express $A_{K}(\rho)$ in a MacLaurin expansion around $\rho=0$ to remove the restriction on the lower limit of $\rho$. Such an expansion is not valid because Eq. (14) was derived assuming a strictly intermediate range for $\rho$. Furthermore, the rest of their analysis pertaining to $\Gamma_{11}$ and 
$\Gamma_{22}$, particularly for the strong scintillation limit (characterized by the scintillation index $m_{Z}^{2}=\left\langle\delta I^{2}\right\rangle /\langle I\rangle^{2} \sim$ 1 ), with the exception of asymptotic solutions, is essentially based on numerical integration methods. Our effort, therefore, has been to obtain an analytical solution for $\Gamma_{11}$ over a wider range than the intermediate, while also being closer to the Kolmogorov power spectrum than the Gaussian.

\section{Approximate Structure Function for Kolmogorov Power Density and Rayleigh-Ritz Optimization}

First, we define $(\rho, q)$ to be the transform variables in the transverse space domain and the spatial wavenumber domain, respectively. By assuming cylindrical (polar) geometry, we express the structure function $A(\rho)$ of a medium with a power spectral density $P(q)$ by means of a Fourier-Bessel transform (analogous to circular aperture problems, Goodman $\left.{ }^{13}\right)$ :

$$
A(\rho) / k^{4}=(1 / 2 \pi) \int_{0}^{\infty} q P(q) J_{0}(\rho q) d q,
$$

where $J_{0}(\cdot)$ is the zeroth-order Bessel function of the first kind. It is fairly straightforward to show that both Eq. (3) for the structure function for a Gaussian spectrum and Eq. (14) for a power law spectrum may be derived using Eq. (15).

Substituting Eq. (11) for $P_{K}(q)$ in Eq. (15), we have,

$$
A_{K}(\rho)=\left(B_{K} k^{4} / 2 \pi\right) \int_{0}^{\infty} q \exp \left(-q^{2} / q_{1}^{2}\right)\left(1+q^{2} / q_{0}^{2}\right)^{-\alpha / 2} J_{0}(\rho q) d q .
$$

Closed analytical solutions of Eq. (16) are not available. Hence we shall expand the Gaussian term in Eq. (16) to two terms (which resembles Lerche's approach); however, we shall retain the power law in the denominator.

We thus have, assuming $q \ll q_{1}$,

$$
\begin{aligned}
A_{K}(\rho)= & \left(B_{K^{k}} k^{4} / 2 \pi\right) \int_{0}^{\infty} q\left(1-q^{2} / q_{1}^{2}\right) \\
& \times\left(1+q^{2} / q_{0}^{2}\right)^{-\alpha / 2} J_{0}(\rho q) \mathrm{d} q, \\
= & \left(B_{K} k^{4} / 2 \pi\right)\left(I_{1}-I_{2}\right),
\end{aligned}
$$

where the integrals $I_{1}$ and $I_{2}$ are shown to be ${ }^{14}$

$$
\begin{aligned}
I_{1}= & {\left[q_{0}^{11 / 3} / 2 \Gamma(11 / 6)\right]\left[q_{0}^{-5 / 3} \Gamma(5 / 6)_{1} F_{2}\left(1 ; 1 / 6,1 ; \rho^{2} q_{0}^{2} / 4\right)\right.} \\
& \left.+(\rho / 2)^{5 / 3} \Gamma(-5 / 6)_{1} F_{2}\left(11 / 6 ; 11 / 6,11 / 6 ; \rho^{2} q_{0}^{2} / 4\right)\right], \\
I_{2}= & \left(q_{0}^{11 / 3} / q_{1}^{2}\right)\left(\left(q_{0}^{1 / 3} \Gamma(2) \Gamma(-1 / 6) /[2 \Gamma(1) \Gamma(11 / 6)]\right\}\right. \\
& \times{ }_{1} F_{2}\left(2 ; 7 / 6,1 ; \rho^{2} q_{0}^{2} / 4\right) \\
& \left.+\left[(\rho / 2)^{-1 / 3} \Gamma(1 / 6) / \Gamma(5 / 6)\right]_{1} F_{2}\left(11 / 6 ; 5 / 6,5 / 6 ; \rho^{2} q_{0}^{2} / 4\right)\right) .
\end{aligned}
$$

In Eqs. (18) and (19), $\Gamma(\cdot)$ are gamma functions, and ${ }_{1} F_{2}(\cdot)$ are hypergeometric functions. It is interesting to note that such hypergeometric functions have been derived recently by Baram et al. ${ }^{15}$ for the slope of the covariance function in the context of remote wind sensing in the presence of atmospheric turbulence with a Kolmogorov spectrum. Their analysis appears to be valid in the intermediate range $\left(q_{0} \ll q \ll q_{1}\right)$.

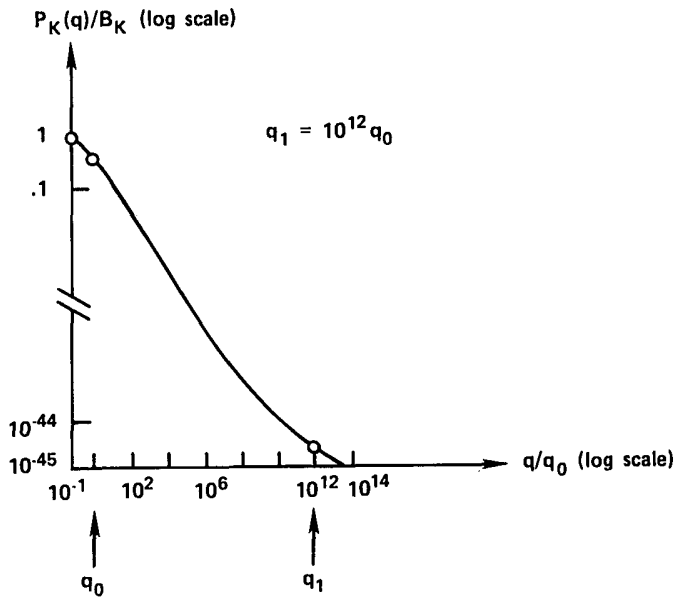

Fig. 1. Kolmogorov power density spectrum with $q_{0} \ll q_{1}$.

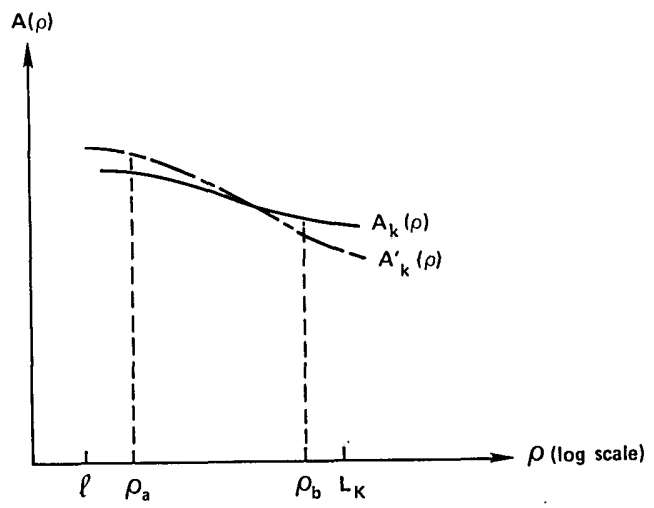

Fig. 2. Structure functions $A_{K}(\rho)$ and $A_{K}^{\prime}(\rho)$.

Using Eqs. (18) and (19) in Eq. (17) and assuming $q_{0}$ $\ll q_{1}$, we finally obtain

$$
A_{K}(\rho) \cong a_{0}+a_{-1 / 3} \rho^{-1 / 3}+a_{5 / 3} \rho^{5 / 3}+a_{2} \rho^{2},
$$

where

$$
\begin{aligned}
a_{0} & =(3 / 5)\left(k^{4} B_{K} / 2 \pi\right) q_{0}^{2}, \\
a_{-1 / 3} & =-2^{1 / 3}[\Gamma(1 / 6) / \Gamma(5 / 6)]\left(q_{0}^{11 / 3} / q_{1}^{2}\right)\left(k^{4} B_{K} / 2 \pi\right), \\
a_{5 / 3} & =(-9 / 50) 2^{1 / 3}[\Gamma(1 / 6) / \Gamma(5 / 6)]\left(q_{0}^{11 / 3}\right)\left(k^{4} B_{K} / 2 \pi\right), \\
a_{2} & =(9 / 10)\left(k^{4} B_{K} / 2 \pi\right) q_{0}^{4} .
\end{aligned}
$$

Equation (20) indicates that $A_{K}(\rho)$ has a singularity at $\rho=0$. However, the expansion of the Gaussian term in Eq. (16) implies that Eq. (20) is valid only for $\rho \gg l$ (the inner scale). Thus the expansion in Eq. (20) does not include $\rho=0$.

To find $\Gamma_{11}$, we must now substitute Eq. (20) for $A_{K}(\rho)$ into Eq. (6). Due to the presence of fractional powers of $\rho$, the resulting equation cannot be analytically solved without considerable difficulty. Since an exact solution for the case where $1-a(\rho)$ has the form $\rho^{2} / L^{2}$ has already been derived, we next optimize Eq. (20) in the form $A_{K}^{\prime}(\rho)$, as shown in Fig. 2, by using a Rayleigh-Ritz technique.

Accordingly, we write 


$$
A_{K}^{\prime}(\rho)=b_{0}+b_{2} \rho^{2}
$$

and minimize the area under the square of the difference from Eq. (20) between two arbitrary points $\rho_{a}$ and $\rho_{b}$ with respect to the unknown coefficients $b_{0}$ and $b_{2}$. Thus letting

$$
\partial I / \partial b_{0}=\partial I / \partial b_{2}=0,
$$

where

$$
I=\int_{\rho_{a}}^{\rho_{b}}\left[A_{K}(\rho)-A_{K}^{\prime}(\rho)\right]^{2} d \rho,
$$

we obtain two simultaneous linear equations in $b_{0}$ and $b_{2}$.

Now from Eq. (22) we have the normalized structure function

$$
a_{K}^{\prime}(\rho)=1+\left(b_{2} / b_{0}\right) \rho^{2} .
$$

Comparing Eq. (25) with the truncated Gaussian expansion in Eq. (7), we may define an effective coherence scale for the Kolmogorov case by

$$
L_{\text {eff }}=\left(-b_{0} / b_{2}\right)^{1 / 2} \text {, }
$$

so that

$$
1-a_{K}^{\prime}(\rho)=\rho^{2} / L_{\text {eff }}^{2} .
$$

The solution of Eq. (6) with Eq. (27) has exactly the same form as Eq. (8) with the only exception being that $L_{\text {eff }}$ replaces $L_{G}$. Thus the above technique has enabled us to express the solution for the two-point twofrequency electric field correlation for the Kolmogorov power density in the same form as that obtained by Lerche for the Gaussian case with two important differences. First, our optimized Kolmogorov solution extends from $\rho_{a}$ to $\rho_{b}$ (where $\rho_{a} \gg l$, and $\rho_{b} \leq 1.94 L_{K}$, where the upper bound on $\rho_{b}$ comes about for reasons explained later), whereas Eq. (8) applies in the range 0 $\leq \rho \leq L_{G}$. Second, the scale lengths of the resulting correlation functions are $L_{\text {eff }}$ and $L_{G}$, respectively, and (as will be shown later) are quite different.

In order that the above optimization procedure yields a meaningful effective coherence length, it is necessary to test if the expression in Eq. (26) is real for all choices of $\rho_{a}$ and $\rho_{b}$. By using the following set of realistic numerical values, $\rho_{a}=10^{10} \mathrm{~cm}, \rho_{b}=10^{18} \mathrm{~cm}$, so that $\rho_{a} \gg l$ and $\rho_{b} \cong L_{K}$, we find that the ratio $\left(-b_{2}\right)$ $\left.b_{0}\right)$, given by

$$
-b_{2} / b_{0}=\frac{\beta_{2}\left(\rho_{b}-\rho_{a}\right)-\beta_{1}\left(\rho_{b}^{3}-\rho_{a}^{3}\right) / 3}{\beta_{2}\left(\rho_{b}^{3}-\rho_{a}^{3}\right) / 3-\beta_{1}\left(\rho_{b}^{5}-\rho_{a}^{5}\right) / 5},
$$

where $\beta_{1}$ and $\beta_{2}$ are functions of $\rho_{a}$ and $\rho_{b}$, becomes

$$
-b_{2} / b_{0}=\frac{-0.0998 q_{0}^{11 / 3} \rho_{b}^{17 / 3}+0.08 q_{q}^{4} \rho_{b}^{6}}{-0.0533 q_{0}^{2} \rho_{b}^{6}+0.004 q_{0}^{11 / 3} \rho_{b}^{23 / 3}} .
$$

Testing the relative signs of the numerator and denominator of Eq. (29), it is fairly simple to show that Eq. (29) is positive for all $\rho_{b}$ values $\left(\rho_{b} \gg \rho_{a} \gg l\right)$, except for a narrow window given by $1.94 / q_{0}<\rho_{b}<4.73 / q_{0}$, or equivalently

$$
1.94 L_{K}<\rho_{b}<4.73 L_{K}
$$

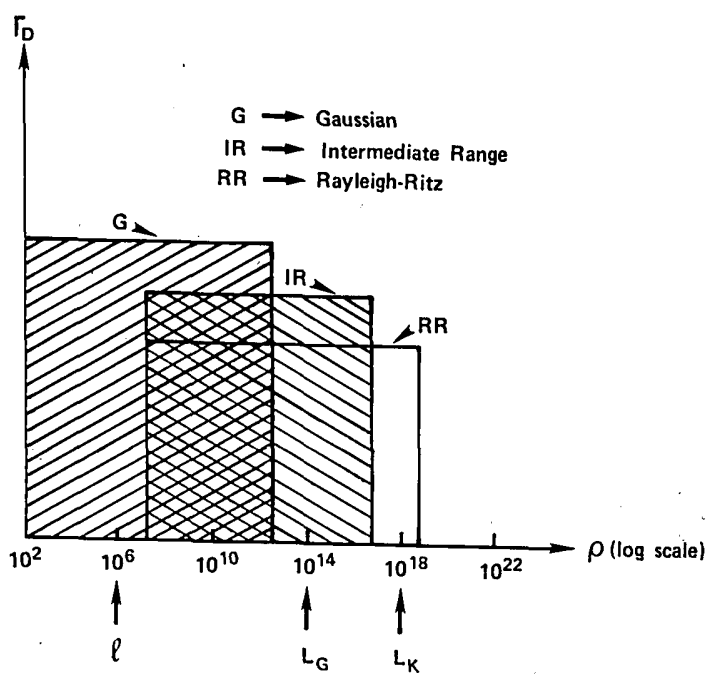

Fig. 3. Domains of validity for (a) approximated Gaussian, (b) intermediate power law, and (c) approximated Kolmogorov.

where the ratio in Eq. (29) is negative, and $L_{\text {eff }}$ becomes imaginary. This window of inadmissible $\rho$ values has been confirmed by programming Eq. (28) in its exact form on a computer. The reason for its existance is not clear. The formalism described by the optimization as in Eqs. (25) and (26) is further restricted to the lower region, $\rho_{b}<1.94 L_{K}$, because $A_{K}^{\prime}(0)$ [or equivalently $b_{0}$ as in Eq. (22)] must be positive in order for $\eta$ and $\xi$ (the normalized $z$ and $\rho$ variables) to be real. While $L_{\text {eff }}$ becomes real again in the region $\rho_{b}>4.73 L_{K}, b_{0}$ is negative in this region, thus resulting in an invalid value for $A_{K}^{\prime}(0)$

To put the various ranges in perspective, we show the domains of validity of the three methods discussed in this paper in Fig. 3. It is clear that while the Gaussian approach includes $\rho=0$, it extends only up to $\rho \ll$ $L_{G}$. The intermediate range results apply (without any analytic solution) in the range $l \ll \rho \ll L_{K}$, and the Rayleigh-Ritz optimization method applies to the range $l \ll \rho \leq \rho_{b}$, where $\rho_{b}$ may approach but not exceed $1.94 L_{K}$.

Finally, to get a feel for the dependence of $\Gamma_{11}(\rho, z)$ on the coordinates $\rho$ and $z$, we have plotted the magnitude of $\Gamma_{D}(\eta, \xi)$ as a function of the normalized variables $\eta$ and $\xi$. This is shown in Fig. 4. For this plot, the following set of values was chosen:

$$
\begin{array}{ll}
R=k_{1} / k_{2}=10, & L_{G}=10^{14} \mathrm{~cm}, \\
\lambda_{1}=0.10 \lambda_{2}=30 \mathrm{~cm}, & L_{K}=10^{18} \mathrm{~cm}, \\
r_{e}=10^{-13} \mathrm{~cm}, & l=10^{6} \mathrm{~cm} . \\
\left\langle\delta N_{e}^{2}\right\rangle=10^{-4} \mathrm{~cm}^{-6}, &
\end{array}
$$

From Fig. 4, we find that the correlation magnitude remains relatively constant over $\sim 4$ orders of magnitude of $\eta$ (at $\xi=0$ ) from $\eta=0$ through $\eta=0.1$ and then drops off fairly rapidly for $\eta>0.1$. The halfwidths along the cross section, on the other hand, decrease by a factor of $\sim 2$ for every decade increase in $\eta\left(\eta \geq 10^{-4}\right)$. We may also note that at $\eta=0$ (the plane of origin of the random medium), $\left|\Gamma_{D}\right|=1$, and the halfwidth is infinite, as expected. To see how the normalized dis- 


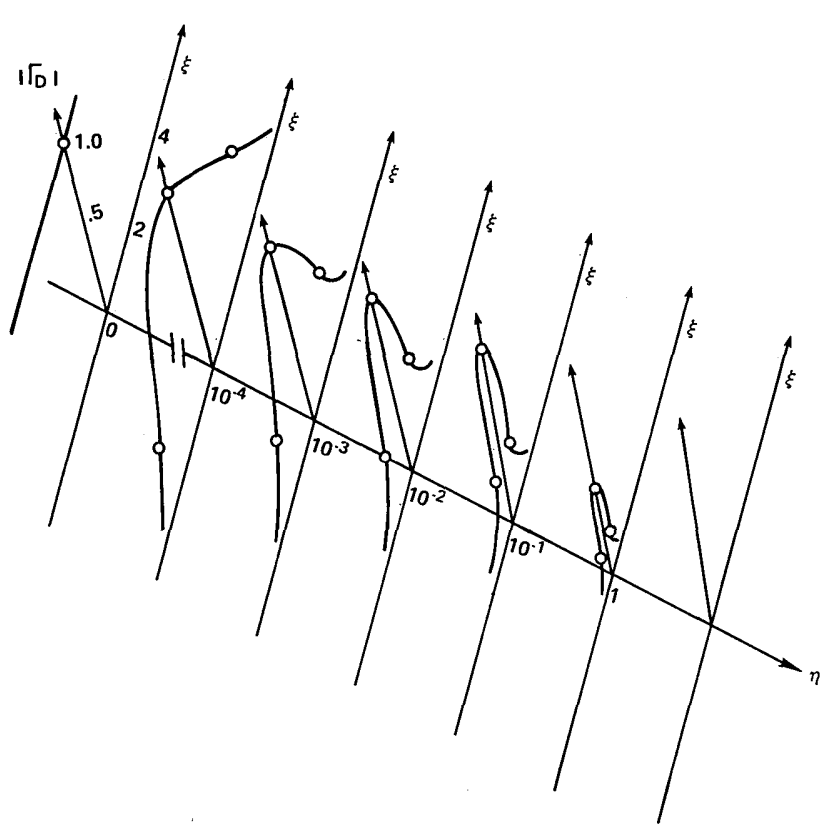

Fig. 4. Spatial evolution of $\left|\Gamma_{D}\right|$ as a function of normalized coordinates of $\eta$ and $\xi$.

tances translate to actual distances in the Gaussian and approximated Kolmogorov cases, we have tabulated the denormalized longitudinal and transverse distances as well as the correlation magnitude and halfwidth in Table I. We find from the table that the correlation persists over distances of $\sim 2$ orders of magnitude higher along the propagation direction and an order of magnitude higher in the transverse direction for the approximated Kolmogorov compared with the Gaussian case. The longitudinal distances over which the electric fields at two points and two frequencies remain correlated are of the order of $10^{21} \mathrm{~cm}$ or less (hence still well below the Fraunhofer limit at $\lambda=30$ $\mathrm{cm})$.

Table I. Magnitude and Effective Width of $\Gamma_{D}$ and Corresponding Transverse and Longitudinal Distances

\begin{tabular}{|c|c|c|c|c|}
\hline$\eta$ & $\begin{array}{c}\left|\Gamma_{D}\right| \\
(\xi=0)\end{array}$ & $\begin{array}{l}\text { Halfwidth } \\
\text { (in } \xi \text { ) }\end{array}$ & $\begin{array}{c}z\left(L_{G}\right) \\
(\mathrm{cm})\end{array}$ & $\begin{array}{c}z\left(L_{\text {eff }}\right) \\
(\mathrm{cm})\end{array}$ \\
\hline $\begin{array}{l}0 \\
1.0 \times 10^{-4} \\
1.0 \times 10^{-3} \\
1.0 \times 10^{-2} \\
1.0 \times 10^{-1} \\
1.0 \times 10^{0}\end{array}$ & $\begin{array}{l}1.0 \\
1.0 \\
1.0 \\
0.9999999 \\
0.9998667 \\
0.5151086\end{array}$ & $\begin{array}{l}\infty \\
3.4 \\
1.9 \\
1.0 \\
0.45 \\
0.2\end{array}$ & $\begin{array}{l}0 \\
4.2829 \times 10^{14} \\
4.2829 \times 10^{15} \\
4.2829 \times 10^{16} \\
4.2829 \times 10^{17} \\
4.2829 \times 10^{18}\end{array}$ & $\begin{array}{l}0 \\
3.5488 \times 10^{16} \\
3.5488 \times 10^{17} \\
3.5488 \times 10^{18} \\
3.5488 \times 10^{19} \\
3.5488 \times 10^{20}\end{array}$ \\
\hline$\xi$ & $\begin{array}{c}\left|\Gamma_{D}\right| \\
\left(\eta=10^{-2}\right) \\
\end{array}$ & & $\begin{array}{c}\rho\left(L_{G}\right) \\
(\mathrm{cm}) \\
\end{array}$ & $\begin{array}{c}\rho\left(L_{\text {eff }}\right) \\
(\mathrm{cm})\end{array}$ \\
\hline $\begin{array}{l}0 \\
1.0 \\
2.0 \\
3.0 \\
4.0 \\
5.0 \\
\end{array}$ & $\begin{array}{l}0.9999999 \\
0.332040 \\
5.0062 \times 10^{-8} \\
0.0 \\
0.0 \\
0.0 \\
\end{array}$ & $\begin{array}{l}0 \\
9.59 \\
1.91 \\
2.87 \\
3.83 \\
4.79\end{array}$ & $\begin{array}{l}28 \times 10^{9} \\
86 \times 10^{10} \\
78 \times 10^{10} \\
71 \times 10^{10} \\
64 \times 10^{10} \\
\end{array}$ & $\begin{array}{l}0 \\
8.7320 \times 10^{10} \\
1.7464 \times 10^{11} \\
2.6196 \times 10^{11} \\
3.4928 \times 10^{11} \\
4.3660 \times 10^{11}\end{array}$ \\
\hline
\end{tabular}

\section{Conclusion}

Although other approximation methods, including Pade and power law, have been used previously, we obtained a structure function which corresponds more closely to the exact Kolmogorov power density spectrum. To construct an analytical solution for the second moment of the electric field, we used the RayleighRitz method, thereby defining an effective coherence scale of fluctuations and extending the range of the valid analytical solution to a distance somewhat higher than the outer scale of fluctuations for the Kolmogorov spectrum. This is an improvement over both the Gaussian spectrum and the intermediate range approximation. Similar techniques may be applied to the evaluation of fourth moments as well.

The authors would like to thank Steven Spangler, University of Iowa, for suggesting this problem.

\section{References}

1. V. I. Tatarski, The Effect of Turbulent Atmosphere (U.S. Department of Commerce, Washington, DC, 1971).

2. R. L. Fante, "Inner-Scale Size Effect on the Scintillations of Light in the Turbulent Atmosphere," J. Opt. Soc. Am. 73, 277281 (1983).

3. R. Dashen, "Path Integrals for Waves in Random Media," J. Math. Phys. 20, 894 (1979).

4. A. Ishimaru, Wave Propagation and Scattering in Random Media (Academic, New York, 1978).

5. V. E. Zuev, "Laser-Light Transmission Through the Atmosphere," in Laser Monitoring of the Atmosphere (Springer-Verlag, Berlin, 1976), Vol. 14, p. 29.

6. K. S. Gochelashvily and V. I. Shishov, "Saturated Fluctuations in the Laser Radiation Intensity in a Turbulent Medium," Sov. Phys. JETP 39, 605 (1974).

7. R. J. Hill and S.F. Clifford, "Modified Spectrum of Atmospheric Temperature Fluctuations and Its Application to Optical Propagation,” J. Opt. Soc. Am. 68, 892-899 (1978).

8. B. Balsley and V. L. Peterson, "Doppler-Radar Measurements of Clear Air Turbulence at $1290 \mathrm{MHz}$," J. Appl. Meteorol. 20, 266 (1981).

9. I. Lerche, "Scintillations in Astrophysics. I. An Analytic Solution of the Second-Order Moment Equation," Astrophys. J. 234, 262 (1979).

10. L. C. Lee and J. R. Jokipii, "Strong Scintillations in Astrophysics. I. The Markov Approximation, Its Validity and Application to Angular Broadening," Astrophys. J. 196, 695 (1975).

11. L. C. Lee and J. R. Jokipii, "Strong Scintillations in Astrophysics. II. A Theory of Temporal Broadening of Pulses," Astrophys. J. 201, 532 (1975).

12. L. C. Lee and J. R. Jokipii, "Strong Scintillations in Astrophysics. III. The Fluctuations in Intensity," Astrophys. J. 202, 439 (1975).

13. J. W. Goodman, Introduction to Fourier Optics (McGraw-Hill, New York, 1968).

14. I. S. Gradshtein and I. M. Ryzhik, Table of Integrals, Series and Products (Academic, New York, 1965).

15. A. Baram, S. Tsadka, Z. Azar, and M. Tur, "Wave Propagation in Turbulent Media: Use of Convergence Acceleration Methods," Appl. Opt. 27, 2145-2149 (1988). 